\title{
Designing a System for Mobile Manipulation from an Unmanned Aerial Vehicle
}

\author{
Christopher M. Korpela, Todd W. Danko and Paul Y. Oh \\ Drexel Autonomous Systems Lab \\ Drexel University \\ Philadelphia, PA \\ cmk325@drexel.edu,twd25@drexel.edu,paul@coe.drexel.edu
}

\begin{abstract}
Due to their significant mobility advantages, UAVs have the potential to perform many tasks in locations that would be impossible for an unmanned ground vehicle to reach. However, most commercially available UAVs currently do not have the required lift to support high performance robotic arms. Recent advances in UAV size to payload and manipulator weight to payload ratios suggest the possibility of integration in the near future. Therefore, we seek to investigate and develop the tools that will be necessary to perform tasks when this becomes a reality. To emulate the UAV, we utilize a six degree-of-freedom gantry crane that provides the complete range of motion of a rotorcraft. Two seven degree-of-freedom manipulators attached to the gantry system perform grasping tasks. Computer vision techniques, including visual servoing, provide target object and manipulator position feedback to the control hardware. To test and simulate our system, we leverage the OpenRAVE virtual environment and ROS software architecture. Because rotorcraft are inherently unstable, introduce ground effects, and experience changing flight dynamics under external loads, we seek to address the difficult task of maintaining a stable UAV platform while interacting with objects using multiple, dexterous arms. As a first step toward that goal, this paper describes the design of a system to emulate highly dexterous manipulators on a UAV.
\end{abstract}

Index Terms-mobile manipulation, unmanned aerial vehicle, dexterous arms

\section{INTRODUCTION}

Unmanned Aerial Vehicles (UAVs) continue to play an increasing role in homeland security, military operations, and civilian-sector applications. Used primarily for surveillance and target acquisition, these vehicles can move quickly and avoid obstacles that would otherwise slow or impede the movement of a ground vehicle. Rotary-wing aircraft such as helicopters and quadrotors can navigate through narrow passages where fixed-winged aircraft cannot. Rotorcraft can also hover and stare, which is helpful when interacting with stationary objects. Some rotorcraft are even able to perch to prolong battery life and conduct surveillance. Advances in navigation, localization (even where GPS is unavailable), and obstacle detection and avoidance have enabled greater autonomy for UAVs. Stable flight and strong hover control are important aspects in autonomous UAV operations. Most of the research to date has focused on autopilots, pilotaircraft interaction, and crash avoidance. Generally, the focus

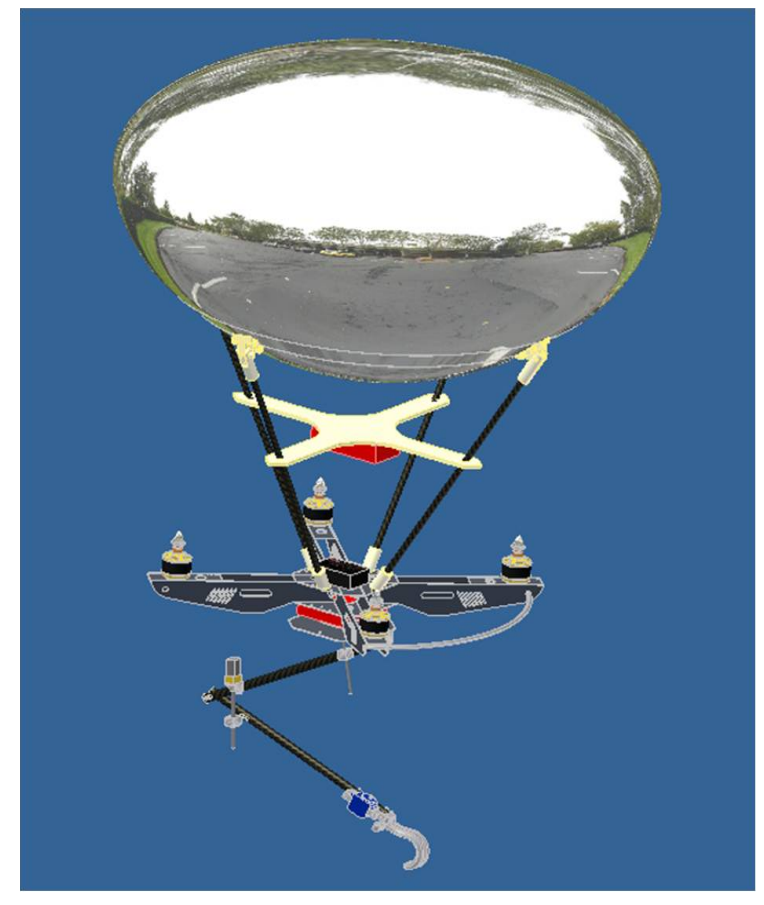

Fig. 1. Hybrid Quadrotor-Blimp Prototype

of previous research is to avoid the ground rather than interact with it, which is our interest.

In addition to autonomous indoor and outdoor navigation, the ability for air vehicles to manipulate a target or carry objects they encounter could greatly expand the types of missions achievable by unmanned systems. Typical applications could be diffusing an Improvised Explosive Device (IED), performing maintenance on a bridge or building, or clearing rubble in a hazardous area. A UAV capable of manipulating its environment could also remove obstacles that are blocking the view of a target or perch to conserve power. However, UAVs currently lack the manipulator arms that many ground vehicles incorporate into their chassis. Ground robots typically focus on mobility and sacrifice manipulation or vice-versa. In our design, we focus on the manipulator and target object interaction while emulating most of the UAV functionality using a 


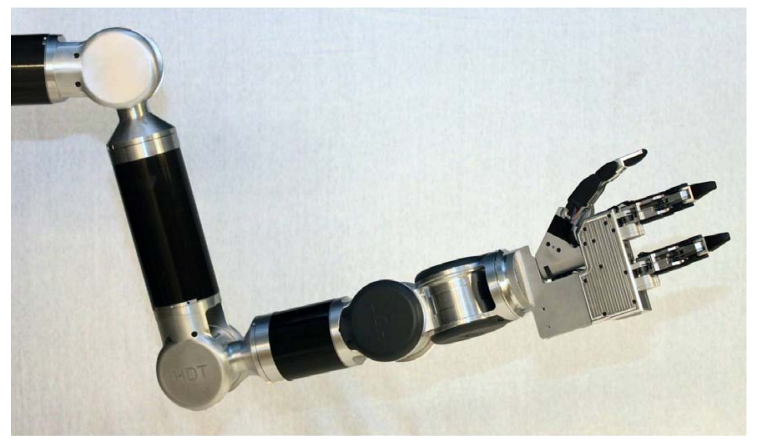

Fig. 2. MK1 Robotic Arm

controlled gantry system. A concept vehicle shown in Figure 1 being constructed by mechanical and electrical engineering students at Drexel University. The elliptical structure at the top of the vehicle is an envelope containing a lighter than air gas and provides two key benefits; it increases the vehicle's moment of inertia while reducing weight, and shifts the center of gravity downward for improved stability. Beneath the gas envelope is a light weight quadrotor airframe to provide equalibrium, the primary means of lift and maneuvering forces. Finally, the 2-DOF manipulator system consists of pulleys to move the actuators underneath the quadrotor structure. We envision a next-generation prototype using a RC rotorcraft and multiple, highly dexterous arms.

High performance arms with end effectors typically weigh more than $20 \mathrm{~kg}$, which cannot be supported by most commercially available UAVs. However, recent developments and technological trends suggest that payload capabilities will increase and arm weights will decrease [1]. Therefore, we believe that highly dexterous off-the-shelf manipulators could soon be supported by RC-hobbyist rotorcraft. An example manipulator with promising characteristics is the MK1 Robotic Arm (Figure 2). It can be used for missions such as IED disposal, hazardous material removal, perching, and homeland security applications. With 11 actuated degrees of freedom and 17 articulated joints, this arm (with hand) has near-human dexterity and better than human strength. One of the primary advantages is its light weight - coming in just over $6.5 \mathrm{~kg}$ while yielding a load capacity of over $22 \mathrm{~kg}$. As arm weights are trending downward, we predict that other dexterous arms will drop to the $10 \mathrm{~kg}$ total mass range in the next few years.

Mobile manipulators on aircraft face significantly more difficulties than traditional ground robots performing mobility tasks in concert with manipulation. The most obvious and difficult facet is the lack of a stable platform. Ground vehicles can remain stationary and provide a very stable base during the manipulation process, whereas aerial vehicles almost never have this benefit. In fact, even with very strong hover control, the aerial platform will never be perfectly stable. This error can partly be compensated for through manipulator control, but it still poses a significant limitation. The angle of approach is also severely restricted using an aerial vehicle, which limits both the manipulator's range of motion and the sensors' field of view. Further, the need for landing gear restricts the manipulator workspace as most dexterous arms will require mounting directly to the underbelly of the aerial vehicle. Our gantry system allows us to control these variables, model and observe reactionary forces, and place the focus on the manipulation-UAV platform coordination. However, a future prototype will have to account for these variables along with many other constraints.

In this paper, we describe an early phase towards using an unmanned aerial vehicle to perform mobile manipulation. First, we look at related work in this research area. Next, we discuss our approach and the overall system design. Subsequently, we outline the hardware system, software infrastructure, sensor suite, and simulation environment. We then propose a concept vehicle as a platform that can support multidegree-of-freedom manipulator arms, with a presentation of test and simulation results. Finally, we chart our future work and conclusions.

\section{RELATED WORK}

Highly dexterous manipulators on ground-based systems are of great interest for commercial and military applications due to their ability to interact with their environment. NASA's Robonaut, University of Massachusetts' uBot, and Willow Garage's PR2 all include dual manipulators fixed to a mobile base. There are also many dual arm systems on a fixed base such as DARPA's ARM Robot, Massachusetts Institute of Technology's DOMO, and University of Massachusetts' Dexter robot. Some examples of single arm manipulators on a dynamic base are Massachusetts Institute of Technology's Cardea and HERB. The systems most related to our design include those on a mobile base that must dynamically balance during the manipulation task. Humanoids such as the KAIST HUBO and Honda ASIMO share our challenge of compensating for a constantly changing center of gravity during mobility and manipulation. The Humanoid PIRE (Partnership for International Research and Education) hosted by Drexel University and funded through the National Science Foundation is using full-scale, mini, and virtual HUBO platforms to study bipedal locomotion and grasping [2]. Other systems using the Segway RMP as a mobility platform face similar challenges with changing centers of gravity.

In addition to leveraging work on manipulators attached to ground vehicles, we will utilize advances in UAV technologies. Autonomy for rotary-wing unmanned air vehicles is being studied at numerous universities, research centers, and private companies, which will help stabilize our platform. Advances in materials and electronics have allowed researchers to achieve small form-factors and light weights [3], [4]. There are a number of aerial testbeds to study single and multi-robot coordination and perform algorithm testing [5], [6]. Many laboratories utilize motion capture systems, implementing an array of high-speed cameras in an indoor extrusion chamber. With improvements in mobile manipulation techniques, particularly with ground robots, these methods are now being applied to aerial vehicles as well. The Yale Aerial Manipulator can grasp 


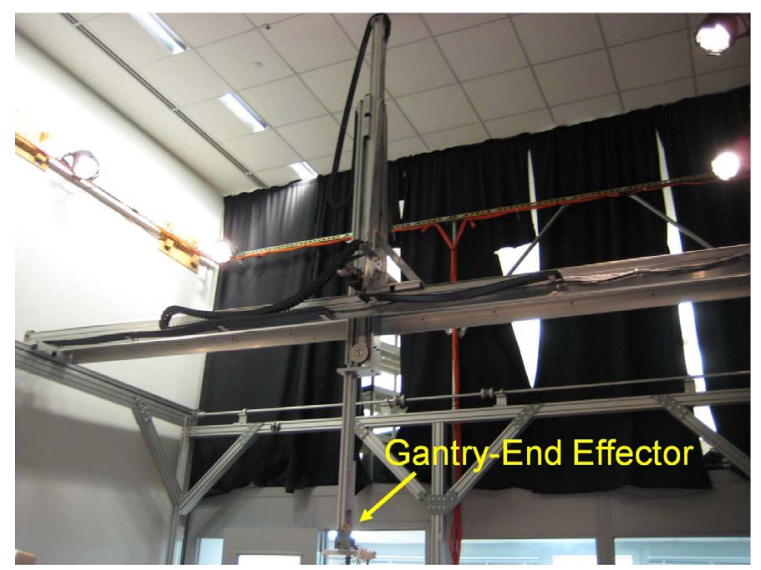

Fig. 3. Systems Integrated Sensor Test Rig (SISTR)

and transport objects using a compliant gripper attached to the bottom of a T-Rex $600 \mathrm{RC}$ helicopter [7]. Researchers at the GRASP Lab at The University of Pennsylvania are using multiple quadrotors to transport payloads in three dimensions using cables [8]. Previous research at Drexel has produced a prototype UAV pickup mechanism with a hook to deliver and retrieve cargo [9]. While there are numerous ground vehicles that use highly dexterous arms, very few if any small or even large UAVs have multiple DOF manipulators mounted to them. To draw a comparison with biology, most UAV manipulators imitate a bird with a beak or claw opening and closing in a 1-DOF movement. Our goal is to integrate a bulbous head with multiple arms similar to an octopus. We aim to leverage the state of the art in ground-based mobile manipulators and apply that to aerial vehicles.

\section{HARDWARE SYSTEM}

\section{A. Mobility}

A six-degree-of-freedom gantry system provides mobility and emulates the UAV in flight. The gantry used is known as the Systems Integrated Sensor Test Rig (SISTR), seen in Figure 3. This hardware-in-the-loop test and evaluation environment is housed at the Drexel Autonomous Systems Lab (DASL). SISTR's motions are controlled through modelreference adaptive control. Real-time sensor data can be fed into a high-fidelity math model of the unmanned aircraft's dynamics. The model generates motion commands that are used to update gantry motions. The net effect is a hardware-inthe-loop test rig that can rapidly and safely test and evaluate UAVs and sensor suites designed to be used in near-Earth environments. The manipulator prototype is mounted on the underbelly of the gantry system similar to the manner in which it would attach to an actual UAV. SISTR is then used to reproduce the velocities and motion of the unmanned aerial vehicle. This configuration allows for testing of the manipulator system in simulated flight conditions [10] such as fog, light, and wind. Previous work has emulated aircraft such as the Raptor, SR-100, and MAKO UAV.

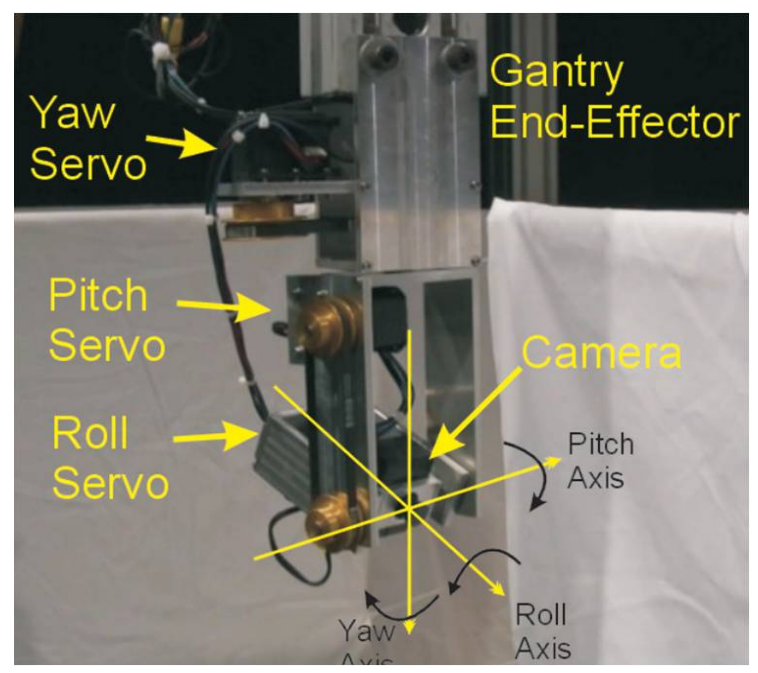

Fig. 4. SISTR's End Effector

The gantry end effector and attached arm move as one unit. The location $(x, y)$, height $z$, yaw $\psi$ where the UAV points, pitch $\theta$, and roll $\varphi$ are all necessary and sufficient to describe its hovering location. We cannot assume any of the orientations to be zero. These variables will change when the manipulator changes position or when leaving the hovering condition and moving to another location. Therefore, the state of the UAV in spatial coordinates can be described as:

$$
s=[x, y, z, \theta, \psi, \varphi]
$$

We utilize the three Euler angles for pitch, yaw, and roll to denote the orientation of the aircraft [11]. The gantry system moves to a desired position and yaw angle while allowing for changes in the pitch and roll angles.

The workspace under the gantry is $5.5 \mathrm{~m} \times 4.25 \mathrm{~m} \times 1.8 \mathrm{~m}$, which provides an ample area for manipulation in an indoor or outdoor environment. SISTR's end effector, shown in Figure 4, is used to represent the location of the UAV in the environment. The aircraft dynamics are handled by the X-Plane flight simulation package [12]. The location and orientation are constantly updated to provide an accurate snapshot of the UAV pose with respect to the ground.

\section{B. Manipulation}

Two Barrett Whole Arm Manipulators (WAM) provide our manipulation capability. There are seven degrees-of-freedom (three in the shoulder, one in the elbow, and three in the wrist). A three-fingered Barrett hand is mounted at the end of the WAM, providing grasping and end effector capabilities. The WAM weighs a total of $27 \mathrm{~kg}$ and can extend to 1 meter when fully deployed. All electronics for the control of the arm are built into the arm and self-contained [13]. The WAM is widely used throughout industry and academia, so there is a broad range of support and research on this device. The control code and interface is open source to permit custom software to interact with the arm. The joints are back-drivable, providing a safety mechanism in the form of compliance. 


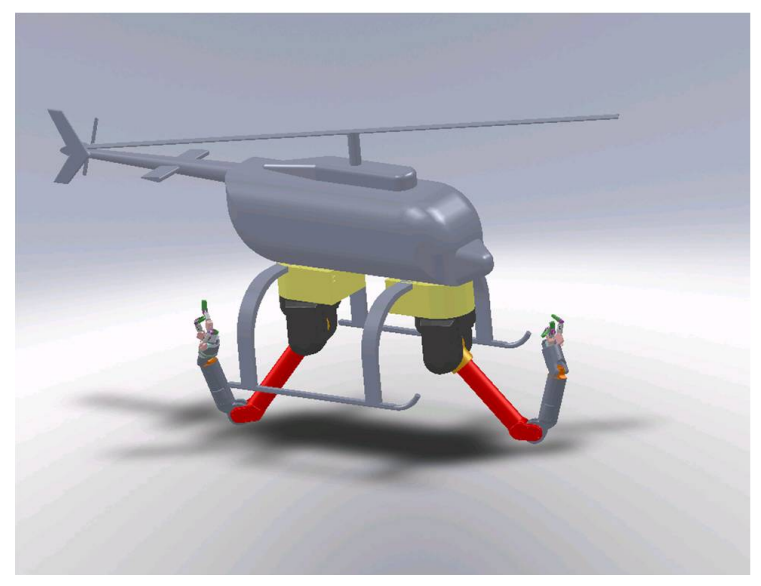

Fig. 5. MM-UAV Concept Drawing

Our design consists of two WAMs that are mounted to the bottom of the gantry crane. We focus on dual arm manipulation and grasping tasks. Figure 5 shows a conceptual drawing of the modeled system. The WAMs are attached perpendicular to the gantry for ease of mounting and to provide the largest range of motion for the arm. However, the stowed configuration must be limited in the z-direction to not interfere with the ability to land and also to limit its impact on flight performance.

\section{Controllers and Power}

All processing and power is tethered through the gantry system. We dedicate one desktop PC for arm control, sensing, and manipulation and another PC for controlling the gantry. Since all of the processing is off-board, we are able to utilize high-end CPUs (quad core processors) that can be constantly powered from an AC wall source. The manipulator, CPUs, and gantry system are connected through an Ethernet switch. Client computers and displays can also easily connect to this configuration for local or remote access. For our prototype design with on-board processing, we utilize micro ATX quad core motherboards with integrated graphics and low-power consumption. At present, we are running all of the control hardware off-board. Power consumption will not be considered until testing begins with our prototype.

\section{Sensor Suite}

Our sensor suite includes various WAM-specific feedback mechanisms (joint encoders and force-torque sensors on the wrist) and external vision systems. For vision, we will utilize an Xbox Kinect camera to facilitate perception of objects in 3D. A Hokuyo LIDAR will provide ranging information back to our control system. Both of these sensors will connect to our PC through a USB interface. Our initial model does not include a camera on the arm itself. The vision system detects the target object to be manipulated and provides feedback of the arm position. Later versions may include an additional camera placement on the wrist of the end effector to provide robustness to occlusions created by the arm. While we do not use a motion capture system, SISTR provides a highly accurate

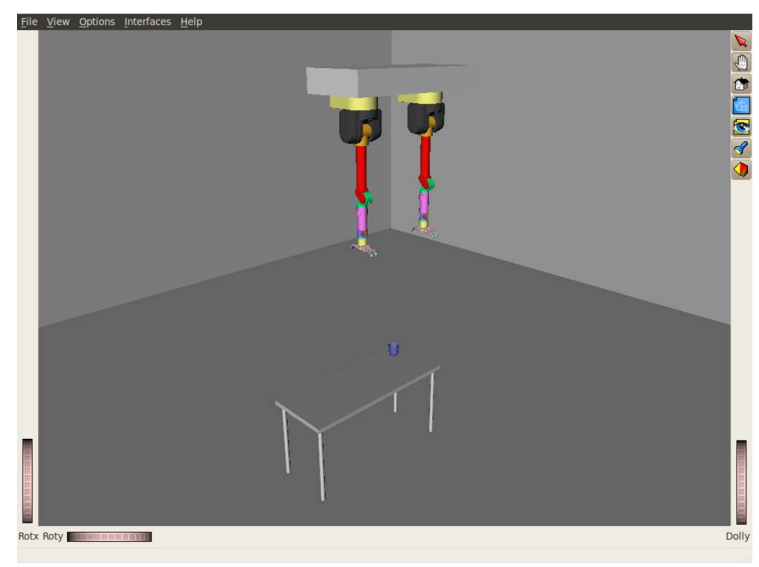

Fig. 6. OpenRAVE Simulation Environment

gantry end effector position. Our current focus is not on UAV localization and mapping.

\section{SOFTWARE INFRASTRUCTURE}

\section{A. Operating Systems}

We utilize Ubuntu Linux (10.04) on our PCs to host ROS (Robot Operating System) navigation and arm manipulation stacks. ROS contains a built-in WAM package that includes forward and inverse kinematic models among other services to allow quick integration with our arm and sensors [14]. All services and data are streamed across ROS to be made available to other services hosted locally or on other networked systems. There is considerable effort in the ROS community to develop manipulation and grasping algorithms for ground robots. Our goal is to leverage and develop these same capabilities for manipulation from an aerial perspective.

\section{B. Simulation Environment}

OpenRAVE, the Open-Source Cross-Platform Robotics Virtual Environment [15], [16], is used for our simulation environment. This simulator allows for easy path planning, collision detection/avoidance, testing, and control in a 3D environment. OpenRAVE handles all of the underlying path formation and trajectory information for the arm and hand. OpenRAVE works best with XML and Python scripts allowing for the easy data input and description of the hardware system. In our environment, we show the WAMs connected to a base representing the gantry crane (Figure 6). The gantry base and attached arms move as one unit in the $x-y-z$ planes along with the ability to change the yaw, pitch, and roll orientations.

\section{Mobile Manipulator UAV CoORdination}

The most difficult and interesting problem that we face while trying to manipulate objects from UAV platforms, lies in the close coordination and control of the manipulation task during hover. Other implementations, as noted in related work, utilize a gripper or hook mechanism with very limited dexterity. A gripper acts more like fingers and the UAV itself as a hand providing most of the manipulation capabilities. 
Our approach establishes a framework to implement dexterous manipulation while hovering with contact only between the end effector and the target object.

As described previously, aerial manipulation is non-trivial. The vehicle must maintain a constant hover while simultaneously gripping an object and rejecting aerodynamic effects [7]. By using highly dexterous arms, our design can perform complex manipulation tasks without the UAV providing the majority of the degrees of freedom. Our initial goal is to characterize the reactionary forces and ground effects the UAV will experience during hover performing manipulation. We will then generate a model to compensate for these forces to provide quasi-stability when in contact with the target. Our objectives are as follows:

- Understand expected moments and loads for a manipulation activity by performing dual arm grasping from a fixed base (mock UAV underbelly).

- Model the UAV reaction to changes in the location of the center of gravity due to arm movements.

- Model the UAV reaction to step input forces during the manipulation task (adaptive gantry system).

- Generate manipulation behaviors to minimize the impact on the UAV (fully integrated dexterous arm and emulated aerial vehicle using the gantry).

- Generate UAV behaviors to establish strong hover control during manipulation tasks.

Our proposed solution to the MM-UAV coordination problem lies with reactive task control and visual servoing. We will use fiducials mounted near the target object and another fixed to the arm end effector. Once the gripper is close enough, we will use force feedback and impedance control to find the target [17]. During this process, the external forces applied to each joint will be measured along with the loads and moments on the base to allow the simulated UAV to properly react.

\section{CONCEPT OF OPERATIONS}

The military currently utilizes teleoperated Unmanned Ground Vehicles (UGVs) for Explosive Ordinance Disposal (EOD) missions. The two primary systems used are the iRobot Packbot and the Foster-Miller TALON. Both systems are small, tracked, battery powered, teleoperated vehicles with a single manipulator arm and gripper. The operator interfaces with the robot via an Operator Controller Unit (OCU) that contains multiple camera displays and joystick control.

Unmanned Aerial Vehicles are also heavily used by the military but primarily for reconnaissance, security, and target acquisition operations. Class I UAVs such as the Honeywell T-Hawk Micro Air Vehicle (MAV) show great promise for use in counter IED missions, but lack the manipulator found on UGVs. Payloads on these small UAVs are currently limited to sensor packages and very minimal lift capabilities. Larger air vehicles, such as the Northrop Grumman MQ-8 Fire Scout and other Type I RC rotorcraft, do have payload capabilities greater than $20 \mathrm{~kg}$, but are either not commercially available or are too expensive [18].

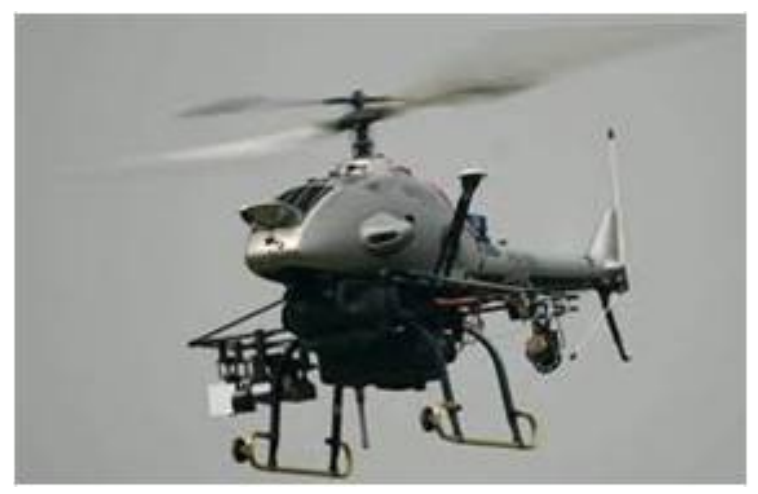

Fig. 7. Yamaha RMAX Helicopter

We envision our Mobile Manipulator UAV system performing the same tasks as existing ground robots, but with a higher degree of autonomy. A possible scenario would involve a rotorcraft such as the Yamaha RMAX (Figure 7) with two Barrett WAMs mounted underneath. The RMAX is over 3 meters in length and can support a $20 \mathrm{~kg}$ payload. The helicopter carries an on-board inertial navigation system providing data on its position $(x, y, z)$, orientation $(\theta, \psi, \varphi)$, velocity, and angular velocities.

With the arm retracted, the landing skids are fully functional for vertical take-off and landing. The helicopter can quickly move to the target area containing an IED. Hovering overhead, the manipulator can perform a diffusing task or simply emplace an explosive charge to destroy the IED.

\section{RESUlts AND Discussion}

We have built a simulation environment for a dual arm UAV manipulator system in OpenRAVE. We chose to perform a pick and place of a cylinder, as it represents one of the more common target object geometries for ground robots and humanoids. Some tests move the cylinder from one surface to another such as a shelf or cupboard. We pick up the cylinder from a table, rotate at the base of the arm, and place the cylinder on the same table at a different location. During the task, the loads and moments on the static base are recorded.

The scenario is kept simple for a number of reasons. First, we are focused on the first task defined in Section V, which is to perform manipulation from a static base. The complexity of the task is not as important as our ability to model the reactionary forces placed on the UAV. A near term goal is to model and replicate reactive forces seen by the UAV in our gantry system. The movement of the arm will constantly alter the center of gravity, and we must simulate these changes in our OpenRAVE environment and measure them on the physical structure. Another significant constraint is the limited field of view for our sensor package. Most tasks will be performed when the aircraft is directly above the target object. With dual manipulators, the aircraft-mounted sensors will be partly occluded during manipulation. We intend to develop sensors to mount further down the arm body to reduce this blockage. Another challenging consideration is the ground 
effect caused by a rotorcraft. Wing in ground effect is the most notable, and the rushing air caused by the rotor could potentially disrupt the target object or stir up sensor occluding debris when moving to a hover position. We intend to model airflow and the effects of rotor wash on the ground as the aircraft changes position.

Our initial experiments involve a stable base and fixed manipulators attached to the ceiling of our OpenRAVE environment. During the pick and place task, we seek to record the moments and loads placed on the fixed base in the simulation environment. Characterizing the worst case load and moment during the full range of manipulator motion is one of our primary objectives. We will map how the center of gravity changes based on the varying positions and pose of the manipulator.

\section{CONCLUSIONS}

We have presented the initial design of a system to simulate an autonomous unmanned aerial vehicle using a mobile manipulator. Our ultimate goal is to build a working prototype of a Type I UAV that can support highly dexterous arms and accompanying sensor package. This vehicle will be able to perform autonomous grasping tasks in a manner similar to that of ground vehicles. By leveraging existing work in grasp planning, mobility, off-the-shelf manipulators, and computer vision techniques, we expect to field a prototype in the next 2 to 3 years. Where much of the focus in mobile manipulation has been with teleoperated ground-based systems, we aim to push the field in the underdeveloped area of autonomous aerial mobile manipulation.

\section{ACKNOWLEDGMENTS}

Chad Kessens from Army Research Labs provided valuable insight, editing, and concept drawings. We would also like to thank the MM-UAV senior design team (Bryan Kobe, Clayton McNeil, and Robert Pisch) for their CAD prototype sketch.

\section{REFERENCES}

[1] http://www.hdtglobal.com/services/robotics/MK1-robotic-arm/.

[2] http://dasl.mem.drexel.edu/pire/.

[3] D. Pines and F. Bohorquez, "Challenges facing future micro-air-vehicle development," AIAA Journal of aircraft, vol. 43, no. 2, pp. 290-305, 2006.

[4] B. Hein and I. Chopra, "Hover performance of a micro air vehicle: Rotor at low reynolds number," Journal of the American Helicopter Society, vol. 52, no. 3, pp. 254-262, July 2007.

[5] N. Michael, D. Mellinger, Q. Lindsey, and V. Kumar, "The GRASP Multiple Micro-UAV Testbed,' Robotics \& Automation Magazine, IEEE, vol. 17 , no. 3 , pp. 56-65, 2010.

[6] G. Hoffmann, D. Rajnarayan, S. Waslander, D. Dostal, J. Jang, and C. Tomlin, "The Stanford testbed of autonomous rotorcraft for multi agent control (STARMAC)," in Digital Avionics Systems Conference, Salt Lake City, UT, 2004.

[7] P. Pounds and A. Dollar, "Hovering Stability of Helicopters with Elastic Constraints," in Proceedings of the 2010 ASME Dynamic Systems and Control Conference, 2010.

[8] D. Mellinger, M. Shomin, N. Michael, and V. Kumar, "Cooperative Grasping and Transport using Multiple Quadrotors," in Distributed Autonomous Robotic Systems, Lausanne, Switzerland, 2010.

[9] N. Kuntz and P. Oh, "Towards Autonomous Cargo Deployment and Retrieval by an Unmanned Aerial Vehicle Using Visual Servoing," in Proceedings of 2008 ASME Dynamic Systems and Controls Conference, 2008.

[10] V. Narli and P. Oh, "Hardware-in-the-loop test rig to capture aerial robot and sensor suite performance metrics," in International Conference on Intelligent Robots and Systems, Beijing, China, 2006.

[11] A. Ng, H. Kim, M. Jordan, and S. Sastry, "Autonomous helicopter flight via reinforcement learning," Advances in Neural Information Processing Systems, MIT Press, 2004.

[12] J. Hing, K. Sevcik, and P. Oh, "Improving unmanned aerial vehicle pilot training and operation for flying in cluttered environments," in International Conference on Intelligent Robots and Systems, St. Louis, MO, 2009.

[13] http://www.barrett.com/robot/products-arm-specifications.htm.

[14] M. Quigley, B. Gerkey, K. Conley, J. Faust, T. Foote, J. Leibs, E. Berger, R. Wheeler, and A. Ng, "ROS: an open-source Robot Operating System," in International Conference on Robotics and Automation, 2009.

[15] R. Diankov, "Automated construction of robotic manipulation programs," Ph.D. dissertation, Carnegie Mellon University, Robotics Institute, August 2010.

[16] R. Diankov and J. Kuffner, "Openrave: A planning architecture for autonomous robotics," Robotics Institute, Pittsburgh, PA, Tech. Rep. CMU-RI-TR-08-34, July 2008.

[17] B. Hamner, S. Koterba, J. Shi, R. Simmons, and S. Singh, "An autonomous mobile manipulator for assembly tasks," Autonomous Robots, vol. 28, no. 1, pp. 131-149, 2010.

[18] G. Cai, B. Chen, and T. Lee, "An overview on development of miniature unmanned rotorcraft systems," Frontiers of Electrical and Electronic Engineering in China, vol. 5, pp. 1-14, 2010. 